\title{
Defensins and the convergent evolution of platypus and reptile venom genes
}

\author{
Camilla M. Whittington, ${ }^{1}$ Anthony T. Papenfuss, ${ }^{2}$ Paramjit Bansal, ${ }^{3}$ Allan M. Torres, ${ }^{4}$ \\ Emily S.W. Wong, ${ }^{1}$ Janine E. Deakin, ${ }^{5}$ Tina Graves, ${ }^{6}$ Amber Alsop, ${ }^{5}$ \\ Kyriena Schatzkamer, ${ }^{6}$ Colin Kremitzki, ${ }^{6}$ Chris P. Ponting, ${ }^{7}$ Peter Temple-Smith, ${ }^{8,9}$ \\ Wesley C. Warren, ${ }^{6}$ Philip W. Kuchel, ${ }^{3}$ and Katherine Belov ${ }^{1,10}$
}

${ }^{1}$ Faculty of Veterinary Science, University of Sydney, Sydney, New South Wales 2006, Australia; ${ }^{2}$ Bioinformatics Division, The Walter and Eliza Hall Institute of Medical Research, Parkville, Victoria 3050, Australia; ${ }^{3}$ School of Molecular and Microbial Biosciences, University of Sydney, Sydney, New South Wales 2006, Australia; ${ }^{4}$ Nanoscale Organisation and Dynamics Group, College of Health and Science, University of Western Sydney, Sydney, New South Wales 1797, Australia; ${ }^{5}$ Australian Research Council (ARC) Centre for Kangaroo Genomics, Research School of Biological Sciences, The Australian National University, Canberra, Australian Capital Territory 0200 Australia; ${ }^{6}$ Genome Sequencing Center, Washington University School of Medicine, St. Louis, Missouri 63108, USA; ' Department of Human Physiology, Anatomy and Genetics, Medical Research Council (MRC) Functional Genetics Unit, University of Oxford, Oxford OX1 3QX, United Kingdom; ${ }^{8}$ Centre for Reproduction and Development, Monash Institute of Medical Research, Clayton, Victoria 3168 Australia; ${ }^{9}$ Department of Zoology, University of Melbourne, Parkville, Victoria 3010 Australia

\begin{abstract}
When the platypus (Ornithorhynchus anatinus) was first discovered, it was thought to be a taxidermist's hoax, as it has a blend of mammalian and reptilian features. It is a most remarkable mammal, not only because it lays eggs but also because it is venomous. Rather than delivering venom through a bite, as do snakes and shrews, male platypuses have venomous spurs on each hind leg. The platypus genome sequence provides a unique opportunity to unravel the evolutionary history of many of these interesting features. While searching the platypus genome for the sequences of antimicrobial defensin genes, we identified three Ornithorhynchus venom defensin-like peptide (OvDLP) genes, which produce the major components of platypus venom. We show that gene duplication and subsequent functional diversification of beta-defensins gave rise to these platypus OvDLPs. The OvDLP genes are located adjacent to the beta-defensins and share similar gene organization and peptide structures. Intriguingly, some species of snakes and lizards also produce venoms containing similar molecules called crotamines and crotamine-like peptides. This led us to trace the evolutionary origins of other components of platypus and reptile venom. Here we show that several venom components have evolved separately in the platypus and reptiles. Convergent evolution has repeatedly selected genes coding for proteins containing specific structural motifs as templates for venom molecules.
\end{abstract}

[Supplemental material is available online at www.genome.org.]

The monotremes have features that place them definitively within the class Mammalia but are unique in laying eggs rather than giving birth to live young. The gestation period of the platypus probably lasts between 15 and $21 \mathrm{~d}$ (Holland and Jackson 2002). The incubation period for eggs is thought to be only 6-10 d (Grant 1995), so at hatching, the nestlings are only about 15 mm long (Grant 1995; Manger et al. 1998) and comparable in development to a newborn marsupial (Manger et al. 1998). Hatchlings remain in the burrow for about $130 \mathrm{~d}$ (Holland and Jackson 2002), during which time they suckle from maternal "milk patches" (Grant 1995). Milk-derived antimicrobial peptides play an important role in immune protection of marsupial young (B.G. Cocks, pers. comm.) and could also protect platypus hatchlings.

To gain more insight into the basic biology of the platypus immune system, we searched the platypus genome for defensin

\footnotetext{
${ }^{10}$ Corresponding author.

E-mail kbelov@vetsci.usyd.edu.au; fax 61-2-9351-3957.

Article published online before print. Article and publication date are at http:// www.genome.org/cgi/doi/10.1101/gr.7149808. Freely available online
} through the Genome Research Open Access option. genes that may play a role in protecting altricial platypus young. Defensins are cysteine-rich peptides with antimicrobial properties that have wide distribution, having been identified in plants, insects, and vertebrates (Patil et al. 2005). Animal defensins possess a distinctive pattern of six cysteine residues, and are categorized into alpha-, beta-, and theta-defensins according to the particular arrangement of disulfide bonds between cysteine residues (Lehrer and Ganz 2002). This same pattern of cysteine interconnections is observed in some components of platypus venom called the OvDLPs ( Ornithorhynchus venom defensin-like peptides).

Male platypuses possess a hollow spur on each hind leg, which is connected via a duct to a venom gland (Grant 1995). Collectively, this is known as the crural system. The platypus venom gland is seasonally active and secretes venom only during the short annual breeding season (Temple-Smith 1973). The venom has mild proteolytic activity and, in laboratory experiments, causes cutaneous anaphylaxis in rabbits and rapid death in mice after intravenous doses of $75-90 \mathrm{mg} / \mathrm{kg}$ (Temple-Smith 1973). In the nonbreeding season the platypus crural system is not functional: The venom gland regresses, its secretory epithe- 
lium becomes inactive, and males show little spurring activity. Evidence from field observations, including increased spurring behavior and male-directed aggression in the breeding season, a high incidence of spur marks on males (Temple-Smith 1973), and the development of the crural system only in the male, suggest that it has evolved primarily as an offensive weapon for use in conspecific aggression to assert dominance over other male platypuses (Temple-Smith 1973).

The crural system can also be used as a defensive weapon, and the occasional unwary fisherman, zookeeper, or biologist may be spurred when handling male platypuses during the breeding season (Grant 1995). Injection of venom into humans results in immediate and acute pain and swelling, which is not alleviated by normal first aid techniques or drugs such as morphine (Fenner et al. 1992). The unusual nature of the symptoms of platypus envenomation may be due to the complex mixture of many different peptides and proteins in the venom, some of which have unknown function (de Plater et al. 1995; Whittington and Belov 2007). Nineteen different fractions have been found in platypus venom (de Plater et al. 1995; Kourie 1999), but only three have been sequenced: OvDLPs (Torres et al. 1999), C-type natriuretic peptides (OvCNPs) (de Plater et al. 1998), and nerve growth factors (OvNGFs) (partially sequenced by A. Torres and P.W. Kuchel, unpubl.). Despite this previous biochemical and some pharmacological research, our knowledge of platypus venom is still incomplete, and additional research is hampered by difficulties in obtaining venom and tissue samples.

The recent sequencing of the platypus genome (Warren et al. 2008) has provided us with a new opportunity to explore the platypus "venome," as well as its "immunome," and has allowed us to examine the evolutionary origins of OvDLPs and beta-defensins.

\section{Results and Discussion}

\section{Platypus alpha- and beta-defensins}

By searching the platypus genome, we identified six beta- and four alpha-defensin genes. One of these (DEFA4) resembles both an alpha-defensin, in the arrangement of its first pair of cysteines, and a beta-defensin, in its arrangement of cysteine residues four and five (Fig. 1), supporting the suggestion of Liu et al. (1997) that alpha-defensins have evolved via gene duplication from beta-defensins and subsequently diversified. The identification of platypus alpha-defensins, previously identified only in therians (Belov et al. 2007), pushes back their emergence to prior to the divergence of the three extant mammalian lineages 210 million years ago (Woodburne et al. 2003).

\section{Synteny analysis}

Beta-defensin genes are arranged in discrete clusters on vertebrate chromosomes. Chickens have a single cluster of 13 beta-defensin genes (chromosome 3q) (Xiao et al. 2004), opossums possess 37 genes in three clusters (Belov et al. 2007), and dog, human, mouse, and rat have $43,39,52$, and 43 beta-defensin genes, respectively, in four to five clusters (Patil et al. 2005). There is a clear evolutionary relationship between these conserved syntenic groups (Patil et al. 2005), as the genes within syntenic clusters form separate clades in phylogenetic trees (Patil et al. 2005; Belov et al. 2007). Because of the fragmented nature of the assembly of the platypus genome (Warren et al. 2008), we used a combination of phylogenetic analysis and FISH mapping to deduce which syntenic groups the platypus genes belonged to and to investigate the evolution of defensins.

Neighbor-joining and Bayesian phylogenetic analyses (Fig. 2; Supplemental Figs. 7, 8) suggest that all but one of the platypus beta-defensin genes belong to a single conserved synteny group (group A) (Fig. 3). In the platypus, these genes map to a single chromosome $\left(\mathrm{X}_{2}\right)$, which supports the topology obtained in our phylogenetic tree (Figs. 2, 3; Supplemental material 5). The single chicken beta-defensin cluster (Xiao et al. 2004) also shares homology with members of synteny group A, making this conserved beta-defensin cluster the most ancient synteny group. Synteny group A also contains the alpha-defensins, which are believed to have evolved from beta-defensins (Liu et al. 1997; Patil et al. 2004). The platypus data suggest that this synteny group gave rise to additional beta-defensin gene clusters through
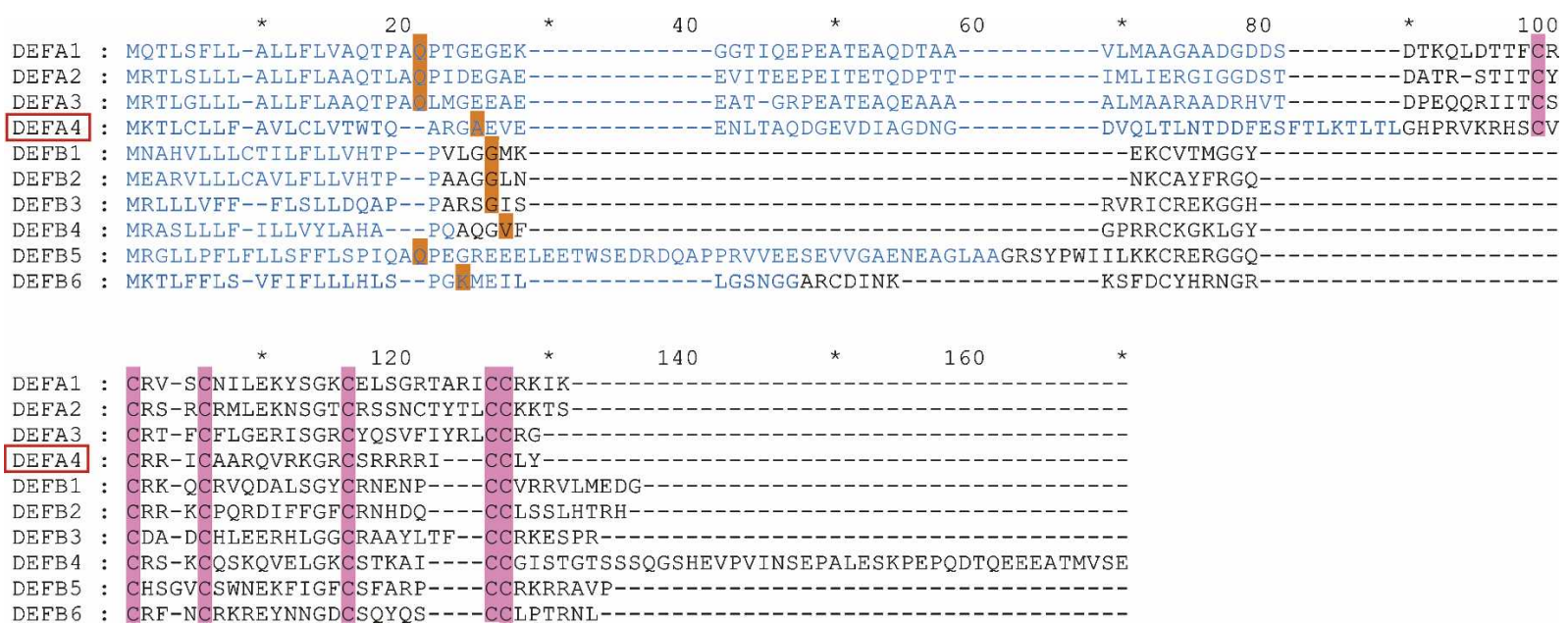

Figure 1. We identified six beta-defensin and four alpha-defensin genes in the platypus genome, shown in alignment. Alternating text color denotes exons, and the first amino acid of the mature peptide is highlighted in orange. Conserved cysteine residues are highlighted in pink. It can be seen that DEFA4 (boxed) has a cysteine spacing that is intermediate between the alpha- and beta-defensins: Like the alpha-defensins, its first two cysteine residues are separated by one amino acid, but the fourth and fifth residues are separated by six rather than nine amino acids. However, DEFA4 does possess an anionic propiece, the net charge of which counterbalances that of its mature defensin domain, which are clear characteristics of alpha-defensins (Liu and Ganz 1995; Hughes and Yeager 1997). 


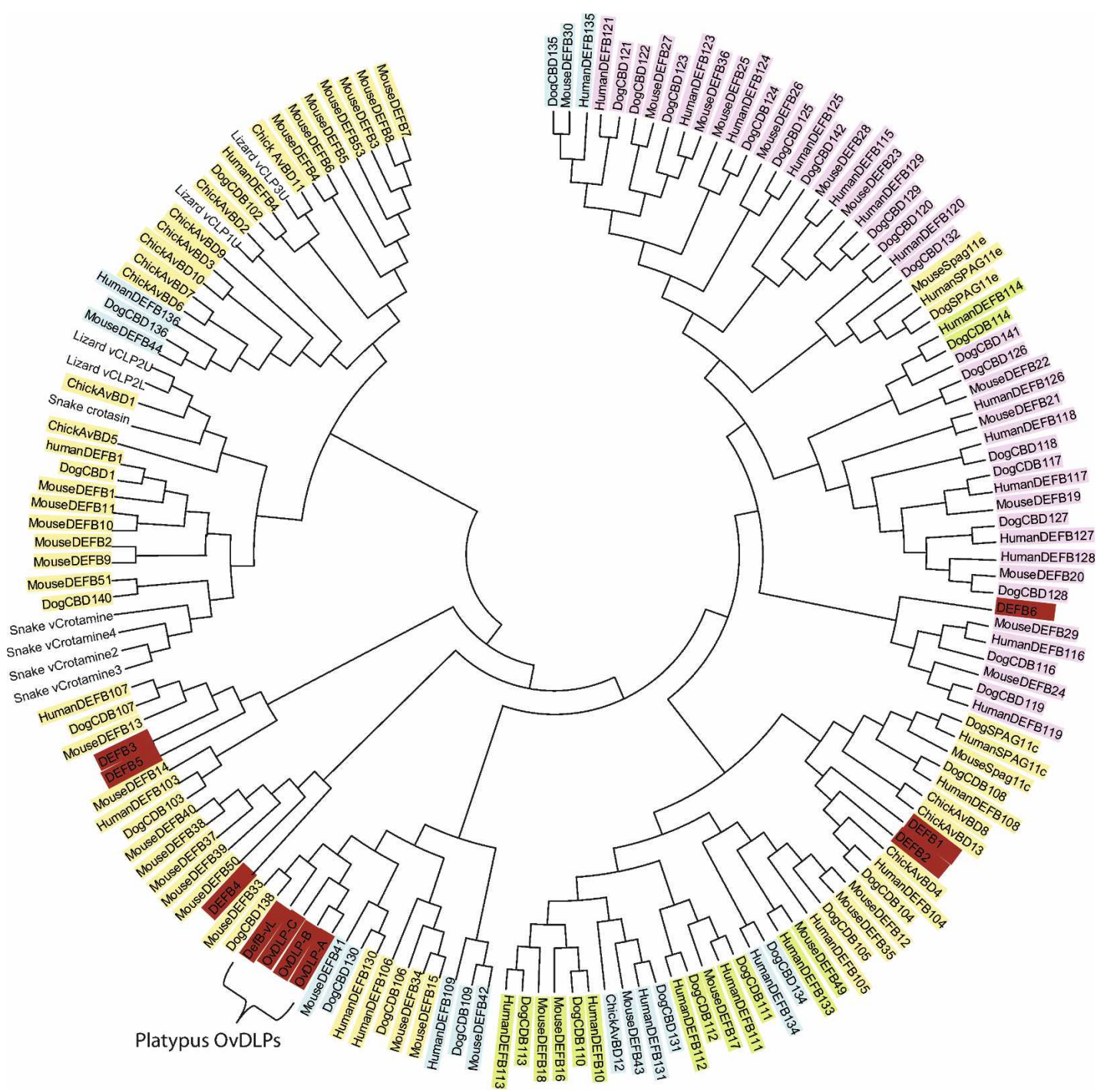

Figure 2. Neighbor joining tree of therian and platypus beta-defensins (DEFB1-6), in red, with chicken beta-defensins (Chick AvBD1-13) and platypus and reptile venom peptides. Chicken and therian sequences are shaded based on whether they belong to synteny groups A (yellow), B (blue), C (green), or D (pink). These synteny groups were determined by Patil et al. (2004) and Belov et al. (2007) based on genomic localization and phylogeny. Platypus $D E F B 1-5$, which we have mapped to chromosome $\mathrm{X}_{2}$, belong to synteny group $\mathrm{A}$, while platypus $D E F B 6$, mapped to chromosome $\mathrm{X}_{1}$, belongs to synteny group D. Similar topologies were recreated using Baysian phylogenetics approaches.

gene duplication and translocation during mammalian evolution. The first beta-defensin cluster (containing DEFB6) to form outside the ancestral cluster appears to be synteny group D, which we have mapped to platypus chromosome $\mathrm{X}_{1}$ (Fig. 3 ). This group arose after the divergence of the birds and mammals but prior to the divergence of the three mammalian lineages. The fact that no members of synteny groups $\mathrm{B}$ and $\mathrm{C}$ were found in the platypus genome suggests that these groups probably arose less than 180 million years ago, after the divergence of the marsupials from the eutherians (Woodburne et al. 2003).

\section{Identification of platypus venom genes}

Searches of the platypus genome using hidden Markov model (HMM) profiles for defensins also identified four other genes encoding putative antimicrobial peptides, with disulfide bonding patterns between cysteine residues that were slightly different from the defensins. Three of these unidentified genes were found to encode defensin-like peptides (OvDLPs), the main peptide components of platypus venom, as they matched partial peptide sequences obtained experimentally (Torres et al. 1999), and the remaining gene appeared to be an intermediate between betadefensins and OvDLPs. It has also previously been suggested that some components of reptile venom may be homologous to betadefensins (Zhao et al. 2001). Using this information, we investigated the genetic organization and evolutionary history of venom genes.

\section{Platypus venom OvDLPs}

The platypus OvDLPs are a family of $5-\mathrm{kD}$ peptides (Torres et al. 1999). The similarity in sequence and structure between betadefensins and OvDLPs suggests that they are evolutionarily related. Significantly, one gene we identified displays characteristics that are a mixture of both peptide families, perhaps representing an evolutionary intermediate between the beta-defensins

\section{Genome Research}

www.genome.org 
A

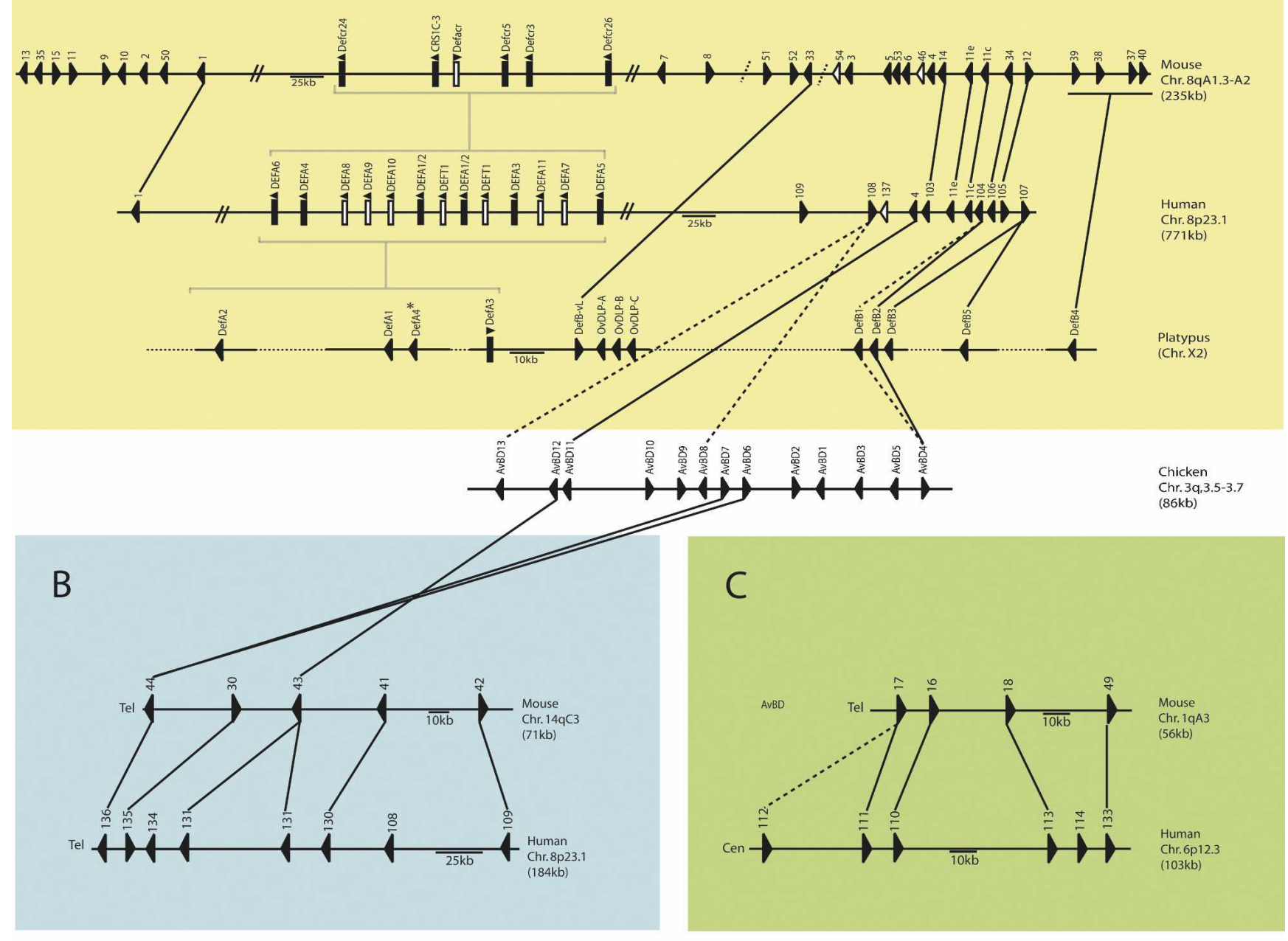

D

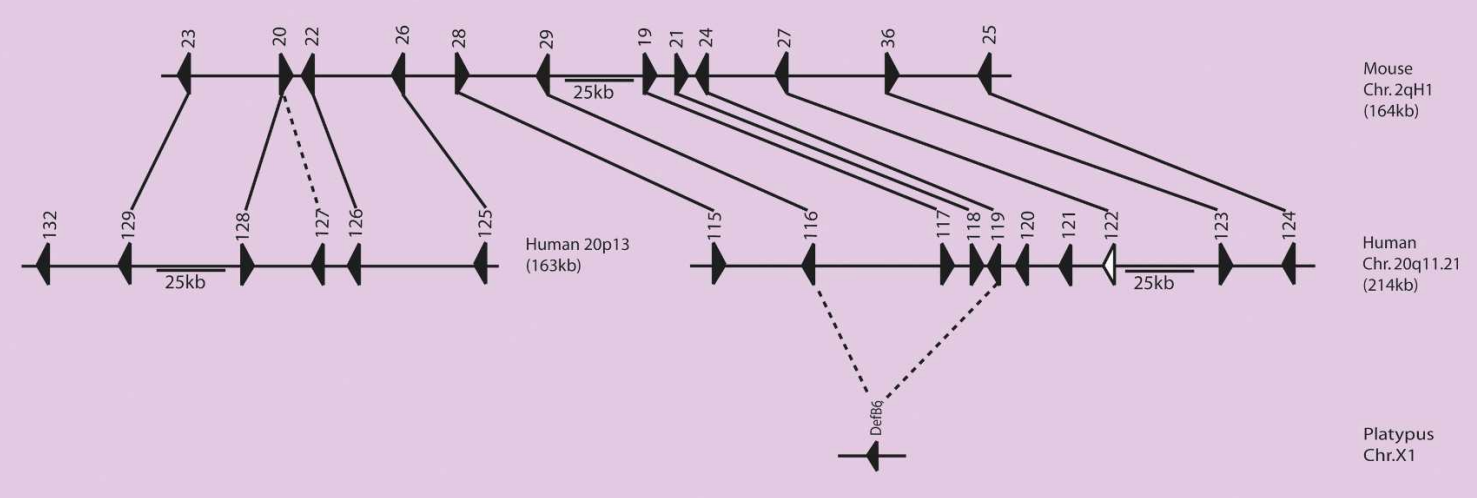

Figure 3. (Legend on next page) 
and OvDLPs. We thus designated this gene DEFB-VL (venom-like beta-defensin). The relatedness of beta-defensins, DEFB-VL, and OvDLPs was confirmed using peptide modeling and gene structure and phylogenetic analysis. There are clear similarities in the structural folds of these peptides (Fig. 4) because of their size and disulfide bond interconnections of the six cysteine residues, and their 1-D NMR spectra also display similarities. DEFB-VL, like the beta-defensins but unlike the OvDLPs (Torres et al. 1999), appears to be antimicrobial (Supplemental material 7). This is due to its N-terminal polyarginine domain, which may allow its interaction with negatively charged phosphate moieties in the cell membranes, and is suggestive of a cell-penetrating domain (Jones et al. 2005). Our fluorescent in situ hybridization (FISH) mapping also supports the hypothesis that OvDLPs are related to betadefensins, as these were all mapped to the same chromosome $\left(\mathrm{X}_{2}\right)$ (Fig. 3; Supplemental material 5).

\section{Features of the defensin and OvDLP genes}

Examination of the intron/exon structure of the beta-defensins, $D E F B-V L$, and OvDLPs showed that they have identical gene structures: two exons, the first encoding a signal peptide and the second encoding the mature peptide containing all six cysteines (Supplemental Fig. 1). Promoter analysis of the defensins and OvDLPs showed that the OvDLP promoters were strikingly similar to one another (89\%-94\% identity). Moreover, searching with known motifs from the TRANSFAC database, CDPCR1 binding sites were predicted in all OvDLPs and three of the defensin promoters. This supports the recent expansion of the OvDLP gene subfamily. These promoters are also present in three of the defensins. All beta-defensins have predicted HNF1A binding sites, and beta-defensins 1-5 have predicted GATA4 binding sites, which suggest tissue specificity (Brahmachary et al. 2006). DEFB$V L$ and beta-defensin 5 have predicted NF-kB binding sites, but only $D E F B-V L$ possesses a glucocorticoid receptor (GR) transcription factor binding site (TFBS). Promoter analysis also revealed putative HNF1A and NF-kB TFBS in DEFB-VL. GR and NF-kB binding sites have previously been found in the promoters of defensins and suggest antimicrobial activity (Brahmachary et al. 2006). Despite the similarity between $D E F B-V L$ and the betadefensins, the spacing of cysteines within the DEFB-VL peptide differs slightly from the beta-defensins, and its amino acid sequence is more similar to that of the OvDLPs than it is to that of the beta-defensins (Supplemental Table 1).

\section{Dating of OvDLP gene duplication}

The OvDLPs and $D E F B$ - $V L$ fall within a pseudoautosomal region on the sex chromosome chain that shares conserved synteny with human chromosome $8 \mathrm{p}$ and chicken chromosome $3 \mathrm{q}$ (Fig. $3)$. We propose that the gene $D E F B-V L$, which lies within $5 \mathrm{~kb}$ of the venom OvDLP genes, gave rise to these genes through gene duplication. We date this duplication to $\sim 192$ million years ago. Subsequent duplications of OvDLPs occurred around 98 and 47

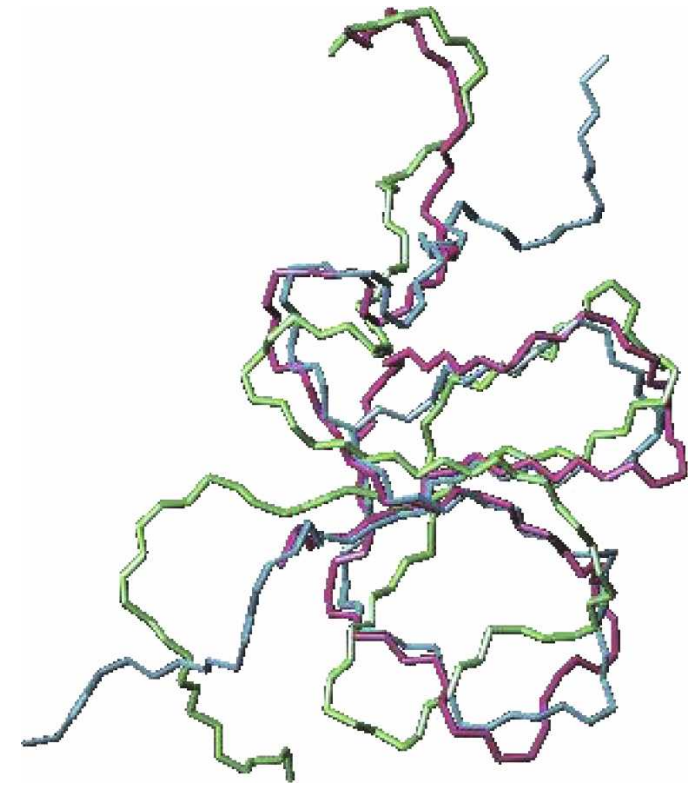

Figure 4. Overlain structural predictions of identified platypus betadefensin and OvDLP peptides produced using LALIGN and MODELLER. The structures of DEFB1 (green), OvDLP-B (pink), and DEFB-VL (blue) peptides show notable similarities, supporting the idea that betadefensins and OVDLPs are evolutionarily related.

million years ago. These venom peptides have thus evolved prior to the divergence of the platypus and the echidna 17-34 million years ago (Gemmell and Westerman 1994; Janke et al. 2002; Belov and Hellman 2003). This suggests that the last common ancestor of echidnas and platypuses may have been venomous. However, although extant male echidnas have a venom delivery system similar to that of the platypus, the crural gland does not migrate to the same position, the spur and its associated os calcaris are less than half the size of the platypus spur-os calcaris complex (Temple-Smith 1973; Temple-Smith and Grant 2001), and the complex cannot be locked in position for spurring (Temple-Smith 1973). This suggests that the echidna crural system may be nonfunctional. There are no published records of echidnas using their spurs, and given that echidnas possess strong spines that provide ample defense against predators, we propose that the crural system, and perhaps its venom peptides, may have been lost during the evolution of the echidna lineage but retained in the platypus lineage for use in defense and conspecific aggression.

\section{Convergent evolution of venom molecules}

The venom of some sauropsid reptiles contains crotamine and crotamine-like peptides (vCLPs) (Torres and Kuchel 2004; Fry et al. 2006), which have a similar disulfide bonding pattern and

Figure 3. Genomic organization of regions of conserved synteny between alpha- and beta-defensins in eutherians, the platypus, and the chicken, based on previously published data (Belov et al. 2007; Patil et al. 2005). Synteny group A, the most ancient beta-defensin synteny group, contains most of the platypus beta-defensins, with synteny group D, containing DEFB6, arising next. Subsequent duplications and translocations have given rise to synteny groups $B$ and $C$ in the later vertebrate lineage. Eutherian genes with labels containing numbers only are beta-defensins. Chicken beta-defensins are labeled as "AvBD," and mouse alpha-defensins (cryptidins) are labeled as "'Defcr." Solid lines link orthologs based on the phylogenetic tree (Fig. 2), and broken lines connect paralogs; pseudogenes are indicated by white arrows; slanted lines indicate breaks in the group; slanted dotted lines indicate a mouse beta-defensin cluster that is not mapped on chromosome 8. An asterisk indicates the putative early alpha-defensin. The fragmented nature of the platypus genome sequence (Warren et al. 2008) meant that, although the platypus alpha- and beta-defensins and the OvDLPs were localized to chromosomes using FISH mapping and phylogenetic analysis, contig order could not be determined and is instead inferred based on synteny.

\section{Genome Research}

www.genome.org 


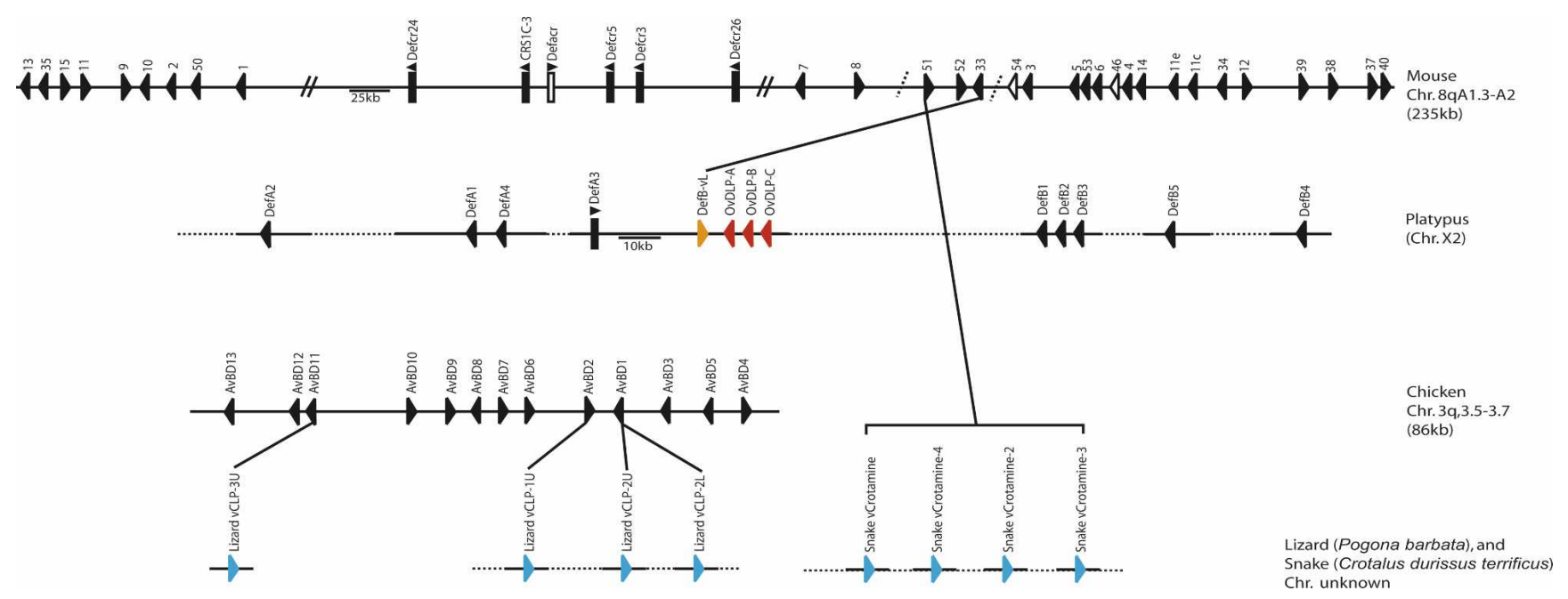

Figure 5. Syntenic relationships of platypus and reptile venom peptides, based on previously published therian synteny (Patil et al. 2005; Belov et al. 2007) and our phylogenetic analysis (Fig. 2). This shows that the platypus OvDLPs and sauropsid reptile vCLPs and crotamines have evolved from different beta-defensin paralogs belonging to the ancestral synteny group A. Genome arrangements of reptile sequences are unknown, so these are inferred based on synteny and connected by dotted lines.

structure to the beta-defensins (Zhao et al. 2001). Our phylogenetic analyses show that these genes appear to have evolved independently from genes belonging to the same ancestral betadefensin synteny group as the platypus OvDLPs (Fig. 5): Lizard vCLPs and chicken beta-defensins 1,2 , and 11 share a common ancestor, and snake venom crotamines and mouse beta-defensin 51 share a common ancestor. The platypus OvDLPs share a common ancestor with mouse beta-defensin 33 .

The independent evolution of both reptile venom crotamines and vCLPs and platypus OvDLPs are not the only examples of venom evolving from antimicrobial peptides. Other groups of antimicrobial proteins, including gamma-thionins and whey acidic protein (WAP) proteins, appear to be similar to animal peptide toxins (Kaplan et al. 2007). Similar selective pressures on antimicrobial peptides (which evolve to target and kill a wide range of rapidly evolving microbes) and venom peptides (which have evolved to specifically cover a wide range of prey) suggest an evolutionary connection between antimicrobial and venom peptides (Kaplan et al. 2007).

In the platypus, antimicrobial peptides are not the only molecules that have given rise to venom peptides. We have also characterized the genes of the only other two sequenced venom components, OvCNP and OvNGF, which have homologs in other species that function in nontoxin roles (Supplemental material 3). Homologous proteins too can be found in reptile venom, and phylogenetic analysis suggests that these venom components may also have originated independently in reptiles and platypuses (Supplemental material 3). Clearly, recruitment of genes involved in nonvenom cellular pathways is a common feature in the evolution of venom, when it is considered that at least 18 molecules in reptile venom have evolved in this way, including acetylcholinesterase, CRISP (cysteine-rich secretory protein), CVF (cobra venom factor), and Factor X (Fry 2005).

\section{Conclusions}

We have identified genes in the platypus for beta-defensins and the earliest known alpha-defensins. We have shown that venom molecules have evolved independently in the platypus and sauropsid reptiles, and we propose that there are specific protein motifs that are preferentially selected for evolution to toxin molecules (Fry 2005). Independent origin of venom molecules appears to coincide with independent origin of venom glands: Snake venom glands evolved from modified salivary glands, whereas platypus venom glands evolved from modified sweat glands (Temple-Smith 1973).

Based on the existence of several species of venomous shrews, it has been proposed that the ancestral mammal may have been venomous (Dufton 1992). Our findings do not support this and nor do those of others who have discovered the fossil of an extinct venomous insectivore that has evidently evolved venom independently from the extant insectivores (Fox and Scott 2005). Instead, we hypothesize that a phylogenetic analysis of shrew venom molecules, impossible at the moment because of the lack of a venomous shrew genome and difficulties in obtaining venom tissues (Dufton 1992), would show an independent origin of these venom molecules from those in platypus and snake venom. However, as our results show, there appears to be a preponderance of certain types of molecules that evolve to have venom function; we speculate that some shrew venom components will be similar to those in reptile and platypus venom.

The availability of the platypus genome has provided an intriguing glimpse into the evolutionary past of mammals and unique insight into the convergent evolution of venom systems by showing that duplication of immune genes has independently given rise to venom molecules several times in the vertebrate lineage. The platypus has much more to offer the field of comparative biology, and given the vast number of useful compounds that have been developed from reptilian venom (Dufton 1992; Fry et al. 2006), platypus venom has considerable potential for the discovery and development of novel therapeutic drugs.

\section{Methods}

\section{Platypus genome searching}

\section{Alpha- and beta-defensins and OvDLPs}

Platypus alpha- and beta-defensins were identified by searching the six-frame translation of the platypus genome using a custom- 
built alpha-defensin profile HMM and the Pfam beta-defensin profile HMM (PF00711) with HMMer (http://hmmer.janelia.org). Genomic sequences that aligned to the HMM were extracted. Peptides that contained stops were discarded.

Next, 267 known mammalian beta-defensins (Patil et al. 2005) were aligned to the platypus genome using TBLASTN (Altschul et al. 1997). The sequences of all HSPs with $E$-values < 1 were extracted and translated. Once again, peptides that contained stops were discarded. The presence of the defensin motif was checked by searching with the alpha- or beta-defensin HMMs and manual inspection. Peptides that did not match the profile HMM or did not contain the six-cysteine motif were discarded.

The HMMer and filtered BLAST data sets were then merged using overlap on the genome. Gene prediction using GenScan (Burge and Karlin 1997) and GenomeScan (Yeh et al. 2001) was performed around each of the features identified in this way. If a defensin motif was found on a contig, gene prediction was performed on the entire contig. For ultracontigs and chromosomes, sequence from the neighborhood was extracted. Predicted proteins were checked for the presence of a signal peptide using SignalP (Bendtsen et al. 2004) and a defensin propeptide using a Pfam model (PF00879).

\section{OvCNPs and OvNGFs}

Partial amino acid sequences obtained by Edman degradation in a protein sequenator for OvCNP (de Plater et al. 1998) and OvNGF (A. Torres and P.W. Kuchel, unpubl.) were obtained. These sequences were then used to mine the platypus genome using a TBLASTN search and HMM profiles for each peptide. Sequences from the neighborhood of matches were extracted, and gene prediction using GenScan (Burge and Karlin 1997) was performed. To assess which genes corresponded to the relevant platypus venom peptide genes, the predicted peptide sequences were used in a BLASTP search against the NR GenBank database. Those returning relevant hits (natriuretic peptides and nerve growth factors, respectively) were then designated putative platypus venom peptide sequences. These were then hand-aligned with the partial amino acid sequences, and nonmatching sequences were removed. Platypus CNPs and NGFs were distinguished from platypus B-type natriuretic peptides and A-type natriuretic peptides, and neurotrophins and brain-derived neurotrophic factors, respectively, by BLASTP searches with the platypus peptides against the human RefSeq protein database on GenBank.

\section{Promoter analysis of OvDLPs and defensins}

We extracted 1200 bp upstream of the beta-defensin and OvDLP translation start sites. Promoter conservation was initially judged by aligning these sequences using MUSCLE (Edgar 2004) and visually inspecting. As the OvDLP promoters appeared to be very highly conserved, we determined the similarity of the betadefensin and OvDLP promoter sequences using PROTDIST from the PHYLIP package (Felsenstein 1989).

Putative transcription factor binding sites were identified in each promoter sequence using MATCH (Kel et al. 2003) and the TRANSFAC database (Matys et al. 2003). Preliminary results were compared within the beta-defensin promoters and the betadefensin and OvDLP promoters. Preliminary results were also compared with known beta-defensin transcription factor binding sites (Brahmachary et al. 2006).

\section{Phylogenetic analysis}

Libraries of similar peptides were assembled for each of the platypus venom peptides. Relevant peptides were identified from BLASTP searches using the full platypus venom peptide se- quences, and sequences identified as being similar to these peptides in the literature were added. The OvCNP-like peptide library consisted of endogenous C-type natriuretic peptides from eutherians, and a Bothrops jararaca endogenous bradykinin potentiating peptide (BPP)-CNP and venom BPP-CNP, with the BPP regions removed from each. A chicken CNP was identified from the chicken genome assembly by a BLAST search of the genome, followed by GenomeScan gene prediction around the hit (coordinates chr21:5752283-57563665), and added to the library. The GenBank accession numbers for these sequences are listed in Supplemental Table 2 . The OvNGF-like peptide library consisted of endogenous beta-NGFs from vertebrates, and snake venom vNGFs (Supplemental Table 3). The OvDLP-like peptide library consisted of lizard venom crotamine-like peptides, snake crotasins, snake venom crotamines (Supplemental Table 4), eutherian beta-defensins (Patil et al. 2005), chicken beta-defensins (Xiao et al. 2004), and platypus beta-defensins identified as above. The alpha-defensin tree consisted of platypus alpha-defensins identified as above, plus eutherian alpha-defensins (Patil et al. 2004), an opossum alpha-defensin (Belov et al. 2007), and chicken betadefensin-12 to root the resulting phylogenetic tree (GenBank accession no. 48976039).

Each of the four peptide libraries (OvCNP-like peptides, OvNGF-like peptides, OvDLP-like peptides, and alpha-defensins) was then aligned using MUSCLE (Edgar 2004). The alignments were then manually improved. The OvDLP-like peptide and alpha-defensin trees were cropped by aligning the full-length sequences in MUSCLE so that the characteristic six cysteines in these peptides aligned. Cropping was then carried out so that only the region containing the characteristic six-cysteine defensin motif remained. A phylogenetic tree for each of the libraries was then constructed in the program MEGA (Kumar et al. 2004) using the Neighbor Joining method (1000 bootstraps, PAM [point accepted mutation] amino acid substitution model, pairwise deletion, homogeneous patterns among lineages, uniform rates of substitution). Bayesian analysis of the OvDLP-like peptide alignments was also carried out using MrBayes 3.1.2 (http:// mrbayes.scs.fsu.edu/) for 1,000,000 generations for the cropped peptide alignment, and 400,000 generations for the full peptide alignment, sampling every 100 generations (Supplemental material 3). Gamma distributed rates were used across sites, and a fixed general time reversible model was used. The results of the Bayesian analysis (Supplemental Figs. 7, 8) confirmed our conclusions that were based on the neighbor-joining phylogenetic analysis.

\section{Dating gene duplication of the OvDLPs}

Duplication dating was done using BEAST (v1.4.5) (http:// beast.bio.ed.ac.uk/) using the relaxed, uncorrelated lognormal molecular clock model (Drummond et al. 2006). We used the Yule process tree prior and calibrated the tree with respect to the molecular divergence date estimates of human and mouse (75 million years ago; Mouse Genome Consortium 2002) and platypus and therians (210 million years ago; Woodburne et al. 2003), under a normal distribution with a 0.5-million-year standard deviation. The length of chain for the Markov chain Monte Carlo (MCMC) process was set at 4,000,000 with parameters logged every 200 generations. Results were analyzed using Tracer (v1.3) (http://beast.bio.ed.ac.uk/). Burn-in was set at 1000, and the final tree was produced using FigTree (v1.0) (http://tree.bio.ed.ac.uk/ software/figtree).

\section{FISH}

Because of the highly fragmented nature of the genome (Warren et al. 2008), FISH was used to map bacterial artificial chromo-

\section{Genome Research}

www.genome.org 
somes (BACs) containing genes of interest. Most procedures, including initiation and maintenance of primary cell cultures, metaphase preparation, $\mathrm{C}_{0} \mathrm{t}-1$ suppressor DNA preparation, labeling of BAC DNA, and FISH were performed as previously described (Alsop et al. 2005). Both single-color and two-color hybridizations were performed. For the latter, BACs were labeled differentially with biotin and digoxygenin and coprecipitated with $\mathrm{C}_{0} \mathrm{t}-1$ DNA. During the fluorescent signal detection steps, anti-digoxygenin and avidin-FITC antibodies were combined in diluent ( $1 \%$ BSA in $4 \times$ SST). Fluorescein isothiocyanate signals were amplified as previously described (Alsop et al. 2005). The optimal slide denaturation time was $\sim 2 \mathrm{~min}$, and 4',6-diamidino-2-phenylindole (DAPI) in Vectashield was used at $1 \mu \mathrm{g} / \mathrm{mL}$.

\section{Modeling and synthesis of DEFB-VL}

\section{DEFB-VL structural prediction}

The DEFB-VL peptide three-dimensional model structure was created from OvDLP-B peptide NMR structure (1zuf) using the MODELLER program (Sali and Blundell 1993). The "very fast modelling protocol" in MODELLER (version 6) was implemented in which DEFB-VL fragments were manipulated to adopt similar structures (secondary and tertiary) to those in the analogous fragments in OvDLP-B peptide NMR structure. Sequence similarities between DEFB-VL and OvDLP-B structures were determined using the LALIGN program (Huang and Miller 1991). A similar modeling scheme was implemented in creating a platypus defensin model from human beta-defensin 1 crystal structure (1iju).

\section{Synthesis of DEFB-VL and OvDLP-B}

The DEFB-VL mature peptide (RRRRRRPPCEDVNGQCQPRGNP CLRLRGACPRGSRCCMPTVAAH) amino acids were assembled manually by stepwise solid-phase synthesis with an in situ neutralization protocol for Boc chemistry (Schnolzer et al. 1992) using benhyrylamine resin $(0.5 \mathrm{mmol}$, loading $0.49 \mathrm{mmol} / \mathrm{g})$. Boc protected amino acids ( $2 \mathrm{mmol}$ ) were coupled using HBTU and diisopropylethyl amine as an activating agent. The side chain protecting groups were $\operatorname{Arg}(\mathrm{Tos}), \operatorname{Asp}(\mathrm{OcHxl}), \operatorname{Asn}(\mathrm{Xan})$, Cys(MeBzl), Gln(Xan), Glu (OcHxl), His(DNP), Ser(Bzl), and Thr(Bzl). Following assembly, the peptide was cleaved from the resin and simultaneously deprotected by treatment with anhydrous HF, using p-cresol and p-thiocresole (50:50) as scavengers at $-2^{\circ} \mathrm{C}$ for 90 min. The crude product was precipitated with cold diethyl ether and then extracted into 50\% aqueous acetonitrile containing $0.05 \%$ trifluoroacetic acid (TFA) and lyophilized.

Purification of the peptide was achieved by preparative RPHPLC (Phenomenex Jupiter $10 \mu \mathrm{C} 18$ column $250 \times 21.20 \mathrm{~mm}$ ) using a linear gradient of $0 \%-50 \%$ acetonitrile in water and $0.1 \%$ TFA over $50 \mathrm{~min}$ at a flow rate of $5 \mathrm{~mL} / \mathrm{min}$. The purified peptide was characterized by mass spectrometry and oxidized by stirring at $4^{\circ} \mathrm{C}$ in an aqueous solution of ammonium hydrogencarbonate (0.1 M, pH 8.2) for $3 \mathrm{~d}$. After completion of oxidation, the $\mathrm{pH}$ of the solution was adjusted between 3-4 pH units and the oxidized peptide isolated using RP-HPLC. It was then characterized by mass spectrometry. The solution phase (90:10::H2O:D2O, 600 $\mathrm{MHz}$ Brucker) NMR spectrum of the fully folded peptide indicated the presence of good structural features that were very similar to OvDLP structures previously reported (Torres et al. 2005).

The same procedure was used to synthesize the mature peptide of platypus OvDLP-B (ImFFEMQACWSHSGVCRDKSERNCK PMAWTYCENRNQKCCEY) (Torres et al. 2005).

\section{$N M R$ on $D E F B-V L$}

All NMR experiments were performed at $25^{\circ} \mathrm{C}$ on a Bruker AVANCE-600 DRX spectrometer using a 5-mm $1 \mathrm{H}$ inverse probe. The 1-D NMR spectra were acquired in phase-sensitive mode using time-proportional phase detection.

\section{Acknowledgments}

We thank the platypus genome consortium for inspiring conversations and the opportunity to work on this project. This work was supported by a grant from the University of Sydney Research and Development Scheme to K.B. and the Australian Research Council to P.K. C.W. is supported by an Australian Postgraduate Award.

\section{References}

Alsop, A., Miethke, P., Rofe, R., Koina, E., Sankovic, N., Deakin, J., Haines, H., Rapkins, R., and Graves, J. 2005. Characterizing the chromosomes of the Australian model marsupial Macropus eugenii (tammar wallaby). Chromosome Res. 13: 627-636.

Altschul, S., Madden, T., Schäffer, A., Zhang, J., Zhang, Z., Miller, W., and Lipman, D. 1997. Gapped BLAST and PSI-BLAST: A new generation of protein database search programs. Nucleic Acids Res. 25: 3389-3402.

Belov, K and Hellman, L. 2003. Immunoglobulin genetics of Ornithorhynchus anatinus (platypus) and Tachyglossus aculeatus (short-beaked echidna). Comp Biochem Physiol A Comp Physiol 136: 811-819.

Belov, K., Sanderson, C., Deakin, J., Wong, E., Assange, D., McColl, K., Gout, A., de Bono, B., Barrow, A., Speed, T., et al. 2007. Characterization of the opossum immune genome provides insights into the evolution of the mammalian immune system. Genome Res. 17: 982-991.

Bendtsen, J., Nielsen, H., von Heijne, J., and Brunak, S. 2004. Improved prediction of signal peptides: SignalP 3.0. J. Mol. Biol. 340: 783-795.

Brahmachary, M., Schonbach, C., Yang, L., Huang, E., Tan, S., Chowdhary, R., Krishnan, S.P.T., Lin, C.Y., Hume, D.A., Kai, C., et al. 2006. Computational promoter analysis of mouse, rat and human antimicrobial peptide-coding genes. BMC Bioinformatics 7: S8. doi: 10.1186/1471-2105-7-S5-S8.

Burge, C. and Karlin, S. 1997. Prediction of complete gene structures in human genomic DNA. J. Mol. Biol. 268: 78-94.

de Plater, G., Martin, R., and Milburn, P. 1995. A pharmacological and biochemical investigation of the venom from the platypus (Ornithorhynchus anatinus). Toxicon 33: 157-169.

de Plater, G., Martin, R., and Milburn, P. 1998. A C-type natriuretic peptide from the venom of the platypus (Ornithorhynchus anatinus): Structure and pharmacology. Comp Biochem Physiol C Comp Pharmacol Toxicol 120: 99-110.

Drummond, A., Ho, S., Phillips, M., and Rambaut, A. 2006. Relaxed phylogenetics and dating with confidence. PLoS Biol. 4: 699-710. doi: 10.1371/journal.pbio.0040088.

Dufton, M. 1992. Venomous mammals. Pharmacol. Ther. 53: 199-215.

Edgar, R. 2004. MUSCLE: Multiple sequence alignment with high accuracy and high throughput. Nucleic Acids Res. 32: 1792-1797.

Felsenstein, J. 1989. PHYLIP-Phylogeny inference package (version 3.2). Cladistics 5: 164-166.

Fenner, P., Williamson, J., and Myers, D. 1992. Platypus envenomation-A painful learning experience. Med. J. Aust. 157: 829-832.

Fox, R. and Scott, C. 2005. First evidence of a venom delivery apparatus in extinct mammals. Nature 435: 1091-1093.

Fry, B. 2005. From genome to 'venome': Molecular origin and evolution of the snake venom proteome inferred from phylogenetic analysis of toxin sequences and related body proteins. Genome Res. 15: 403-420.

Fry, B., Vidal, N., Norman, J., Vonk, F., Scheib, H., Ramjan, S., Kuruppu, S., Fung, K., Hedges, S., Richardson, M., et al. 2006. Early evolution of the venom system in lizards and snakes. Nature 439: 584-588.

Gemmell, N. and Westerman, M. 1994. Phylogenetic relationships within the class mammalia: A study using mitochondrial 12S RNA sequences. J. Mol. Evol. 2: 3-23.

Grant, T. 1995. The Platypus. UNSW Press, Sydney, Australia.

Holland, N. and Jackson, S. 2002. Reproductive behaviour and food consumption associated with the captive breeding of platypus 
(Ornithorhynchus anatinus). J. Zool. 256: 279-288.

Huang, X. and Miller, W. 1991. A time-efficient, linear-space local similarity algorithm. Adv. Appl. Math. 12: 337-357.

Hughes, A.L. and Yeager, M. 1997. Coordinated amino acid changes in the evolution of mammalian defensins. J. Mol. Evol. 44: 675-682.

Janke, A., Magnell, L., Wieczorek, G., Westerman, M., and Arnason, U. 2002. Phylogenetic analysis of 18 S rRNA and the mitochondrial genomes of the wombat, Vombatus ursinus, and the spiny anteater, Tachyglossus aculeatus: increased support for the Marsupionta hypothesis. J. Mol. Evol. 54: 71-80.

Jones, S.W., Christison, R., Bundell, K., Voyce, C.J., Brockbank, S.M.V., Newham, P., and Lindsay, M.A. 2005. Characterisation of cell-penetrating peptide-mediated peptide delivery. Br. J. Pharmacol. 145: $1093-1102$.

Kaplan, N., Morpurgo, N., and Linial, M. 2007. Novel families of toxin-like peptides in insects and mammals: A computational approach. J. Mol. Biol. 369: 553-566.

Kel, A., Gößling, E., Reuter, I., Cheremushkin, E., Kel-Margoulis, O., and Wingender, E. 2003. MATCH: A tool for searching transcription factor binding sites in DNA sequences. Nucleic Acids Res. 31: $3576-3579$.

Kourie, J. 1999. A component of platypus (Ornithorhynchus anatinus) venom forms slow-kinetic cation channels. J. Membr. Biol. 172: $37-45$.

Kumar, S., Tamura, K., and Nei, M. 2004. MEGA3: Integrated software for molecular evolutionary genetics analysis and sequence alignment. Brief. Bioinform. 5: 150-163.

Lehrer, R. and Ganz, T. 2002. Defensins of vertebrate animals. Curr. Opin. Immunol. 14: 96-102.

Liu, L. and Ganz, T. 1995. The pro region of human neutrophil defensin contains a motif that is essential for normal subcellular sorting. Blood 85: 1095-1103.

Liu, L.D., Zhao, C.Q., Heng, H.H.Q., and Ganz, T. 1997. The human beta-defensin-1 and alpha-defensins are encoded by adjacent genes: Two peptide families with differing disulfide topology share a common ancestry. Genomics 43: 316-320.

Manger, P., Hall, L., and Pettigrew, J. 1998. The development of the external features of the platypus (Ornithorhynchus anatinus). Philos. Trans. R. Soc. Lond. B Biol. Sci. 353: 1115-1125.

Matys, V., Fricke, E., Geffers, R., Gössling, E., Haubrock, M., Hehl, R., Hornischer, K., Karas, D., Kel, A., Kel-Margoulis, O., et al. 2003. TRANSFAC: Transcriptional regulation, from patterns to profiles. Nucleic Acids Res. 31: 374-378.

Mouse Genome Consortium. 2002. Initial sequencing and comparative analysis of the mouse genome. Nature 420: $520-562$.

Patil, A., Hughes, A., and Zhang, G. 2004. Rapid evolution and diversification of mammalian alpha-defensins as revealed by comparative analysis of rodent and primate genomes. Physiol. Genomics 20: $1-11$.

Patil, A., Cai, Y., Sang, Y., Blecha, F., and Zhang, G. 2005. Cross-species analysis of the mammalian beta-defensin gene family: Presence of syntenic gene clusters and preferential expression in the male reproductive tract. Physiol. Genomics 23: 5-17.

Sali, A. and Blundell, T. 1993. Comparative protein modeling by satisfaction of spatial restraints. J. Mol. Biol. 234: 779-815.

Schnolzer, M., Alewood, P., Jones, A., Alewood, D., and Kent, S. 1992. In situ neutralization in Boc-chemistry solid-phase peptide synthesis. Rapid, high yield assembly of difficult sequences. Int. J. Pept. Protein Res. 40: 180-193.

Temple-Smith, P. 1973. "Seasonal breeding biology of the platypus, Ornithorhynchus anatinus (Shaw, 1799), with special reference to the male." Ph.D. thesis, Australian National University, Canberra.

Temple-Smith, P. and Grant, T. 2001. Uncertain breeding: A short history of reproduction in monotremes. Reprod. Fertil. Dev. 13: 487-497.

Torres, A. and Kuchel, P. 2004. The beta-defensin-fold family of polypeptides. Toxicon 44: $581-588$.

Torres, A., Wang, X., Fletcher, J., Alewood, D., Alewood, P., Smith, R., Simpson, R., Nicholson, G., Sutherland, S., Gallagher, C., et al. 1999 Solution structure of a defensin-like peptide from platypus venom. Biochem. J. 341: 785-794.

Torres, A., Tsampazi, C., Geraghty, D., Bansal, P., Alewood, P., and Kuchel, P. 2005. D-amino acid residue in a defensin-like peptide from platypus venom: Effect on structure and chromatographic properties. Biochem. J. 391: 215-220.

Warren, W., Hillier, L., Marshall Graves, J.A., Birney, E., Ponting, C.P., Grutzner, F., Belov, K., Miller, W., Clarke, L., Chinwalla, A.T., et al 2008. Genome analysis of the platypus reveals unique signatures of evolution. Nature 453: 175-183.

Whittington, C. and Belov, K. 2007. Platypus venom: A review. Aust. Mammal. 29: 57-62.

Woodburne, M., Rich, T., and Springer, M. 2003. The evolution of tribospheny and the antiquity of mammalian clades. Mol. Phylogenet. Evol. 28: 360-385. 28.

Xiao, Y., Hughes, A., Ando, J., Matsuda, Y., Cheng, J., Skinner-Noble, D., and Zhang, G. 2004. A genome-wide screen identifies a single beta-defensin gene cluster in the chicken: Implications for the origin and evolution of mammalian defensins. BMC Genomics 5: 56. doi: 10.1186/1471-2164-5-56.

Yeh, R.-F., Lim, L., and Burge, C. 2001. Computational inference of homologous gene structures in the human genome. Genome Res. 11: $803-816$

Zhao, C.Q., Nguyen, T., Liu, L.D., Sacco, R.E., Brogden, K.A., and Lehrer, R.I. 2001. Gallinacin-3, an inducible epithelial beta-defensin in the chicken. Infect. Immun. 69: 2684-2691.

Received September 17, 2007; accepted in revised form December 11, 2007.

\section{Genome Research}

www.genome.org 


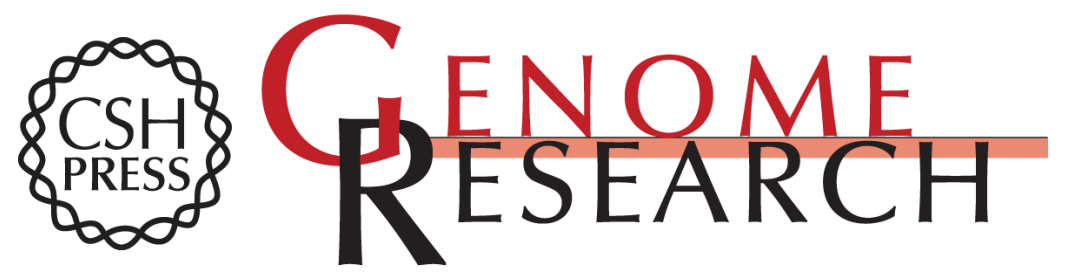

\section{Defensins and the convergent evolution of platypus and reptile venom genes}

Camilla M. Whittington, Anthony T. Papenfuss, Paramjit Bansal, et al.

Genome Res. 2008 18: 986-994 originally published online May 7, 2008

Access the most recent version at doi:10.1101/gr.7149808

\footnotetext{
Supplemental http://genome.cshlp.org/content/suppl/2008/05/08/gr.7149808.DC1

Material
}

Related Content Retroposed SNOfallA mammalian-wide comparison of platypus snoRNAs Jürgen Schmitz, Anja Zemann, Gennady Churakov, et al. Genome Res. June , 2008 18: 1005-1010 Conservation of small RNA pathways in platypus

Elizabeth P. Murchison, Pouya Kheradpour, Ravi Sachidanandam, et al. Genome Res. June, 2008 18: 995-1004 Origin of INSL3-mediated testicular descent in therian mammals Jae-ll Park, Jenia Semyonov, Chia Lin Chang, et al. Genome Res. June , 2008 18: 974-985 Bird-like sex chromosomes of platypus imply recent origin of mammal sex chromosomes Frédéric Veyrunes, Paul D. Waters, Pat Miethke, et al. Genome Res. June , 2008 18: 965-973

References This article cites 47 articles, 6 of which can be accessed free at: http://genome.cshlp.org/content/18/6/986.full.html\#ref-list-1

Articles cited in: http://genome.cshlp.org/content/18/6/986.full.html\#related-urls

Open Access Freely available online through the Genome Research Open Access option.

License Freely available online through the Genome Research Open Access option.
Email Alerting Receive free email alerts when new articles cite this article - sign up in the box at the Service top right corner of the article or click here.

\section{Affordable, Accurate Sequencing.}




\section{Affordable, Accurate} Sequencing.

To subscribe to Genome Research go to: https://genome.cshlp.org/subscriptions 